\title{
PERIOD DOUBLING AND THE LEFSCHETZ FORMULA
}

\author{
BY
}

JOHN FRANKS ${ }^{1}$

\begin{abstract}
This article gives an application of the Lefschetz fixed point theorem to prove, under certain hypotheses, the existence of a family of periodic orbits for a smooth map. The family has points of periods $2^{k} p$ for some $p$ and all $k \geq 0$. There is a version of the result for a parametrized family $f_{t}$ which shows that these orbits are "connected" in parametrized space under appropriate hypotheses.
\end{abstract}

By far the most useful theorem for proving the existence of fixed points, or, more generally, periodic points, of continuous self-maps of finite-dimensional spaces is the Lefschetz trace formula. It equates the sum of local indices of fixed points with an invariant of the homotopy class of the map. To study periodic, as well as fixed, points, it is necessary to keep track of infinitely many equalities of this sort, one for each iterate of the map $f$ in question. The most efficient bookkeeping scheme for doing this seems to be the Lefschetz zeta function $Z(f)$. This is a rational function of a single variable $t$ which depends only on the homotopy class of $f$ and can be expressed either in terms of homological invariants of $f$ or in terms of the sum of local indices of fixed points of $f^{n}$ for all $n>0$. A precise definition is given in $\S 1$ below.

We want to apply this invariant to prove the existence of infinite families of periodic orbits with periods $2^{n} p$ for some $p$ and all $n>0$, and also to study the way these families appear and disappear as a continuous parameter for the self-map is varied. This last topic was inspired by the result of Alligood and Yorke $[\mathbf{A Y}]$ which showed that in a one-parameter family of area contracting mappings which produce a horseshoe there are infinitely many "period-doubling cascades".

We will consider smooth maps $f: M \rightarrow M$ which have only transversal periodic points (so-called because the graph of $f^{n}, n>0$, is transverse to the diagonal), i.e. we assume that whenever $n>0$ and $f^{n} x=x, D_{x} f^{n}$ does not have 1 as an eigenvalue.

To use the Lefschetz zeta function in the study of bifurcations, we first observe that it is a bifurcation invariant since it depends only on the homotopy class of $f$. Secondly, we recall that $Z(f)$ can be expressed as an infinite product of terms of the form $\left(1 \pm t^{p}\right)$, each one contributed by a single periodic orbit, and we show algebraically in $\S 2$ that one can "separate out" the contribution to such a product of all the terms of the form $\left(1 \pm t^{2^{n}} d\right)$, for a fixed odd $d$. Thus we show that for each odd $d$ the contribution to $Z(f)$ of all these periodic orbits whose least periods are $2^{n} d$ is a bifurcation invariant. This contribution to $Z(f)$, as a formal power series which we denote $Z(f, d)$, can also be computed from homological invariants. This

Received by the editors June 15, 1983 and, in revised form, February 5, 1984.

1980 Mathematics Subject Classification. Primary 58F20; Secondary 55M20.

${ }^{1}$ Research supported in part by NSF Grant MCS 8002177 and the I.H.E.S. 
is a paraphrase of our main theorem, which is stated more precisely and proved in $\S 3$.

The idea of looking at periodic orbits whose periods have a fixed "oddity" $d$ (i.e. whose periods are $2^{k} d$ for some $k \geq 0$ ) is due to Paul Blanchard [B].

We now give three examples of the kinds of applications which are easy corollaries of this theorem.

If $\gamma$ is a transversal periodic orbit of $f$ with least period $p(\gamma)$, then two integers determine its contribution to the Lefschetz zeta function $Z(f)$. Pick some $x \in \gamma$ and let $E^{u}$ be the subspace of $T M_{x}$ spanned by eigenvalues of absolute value greater than one, i.e. $E^{u}$ is the expanding eigenspace. The dimension of $E^{u}$ is called the Morse index of the orbit. We call the orbit $\gamma$ twisted if $D_{x} f^{p(\gamma)}: E^{u} \rightarrow E^{u}$ reverses orientation, and untwisted otherwise.

Let $\mathrm{PO}(f, d)$ denote the set of all periodic orbits of $f$ whose least period is $2^{k} d$ for some $k \geq 0$, and denote by $D^{n}$ the $n$-dimensional disk.

THEOREM A. Let $f: D^{n} \rightarrow \operatorname{int}\left(D^{n}\right)$ be a smooth map with only transversal periodic points. Suppose $d$ is odd and all orbits in $\mathrm{PO}(f, d)$ have odd Morse index. If $\gamma \in \mathrm{PO}(f, d)$ has least period $p$, then for each $k \geq 0$ there is a twisted periodic orbit of $f$ with least period $2^{k} p$. The same conclusion is valid if $d>1$ and all orbits in $\mathrm{PO}(f, d)$ have even Morse index.

DEFINITIONS. We denote by $\operatorname{Fix}(f, n)$ the fixed points of $f^{n}$ (and for simplicity write $\operatorname{Fix}(f)$ for $\operatorname{Fix}(f, 1))$. If $F: M \times I \rightarrow M$ is a one-parameter family of mappings and $\gamma_{a}$ and $\gamma_{b}$ are periodic orbits of $F(\cdot, a)$ and $F(\cdot, b)$, respectively, we say $\gamma_{a}$ and $\gamma_{b}$ are connected via $F$ provided there exist $x \in \gamma_{a}$ and $y \in \gamma_{b}$ such that $(x, a)$ and $(y, b)$ are in the same component of $\operatorname{Fix}(\bar{F}, p)$, where $\bar{F}(x, t)=(F(x, t), t)$ and $p$ is a multiple of the (least) periods of $x$ and $y$. We say $F$ has bounded periodic set provided there is a compact set $K \subset M$ which contains $\operatorname{Fix}(F(\cdot, \mu), n)$ for all $n>0$ and all $\mu \in I$.

In the following theorem (as well as the one above) it is important to note that the hypotheses (and conclusions) only involve points of periods $2^{k} d$ for one fixed odd $d$, provided other periodic points are transversal. The presence of transversal periodic points of other periods has no effect.

THEOREM B. Let $F: M \times I \rightarrow M$ be a continuous function with bounded periodic set such that $f_{0}=F(\cdot, 0)$ and $f_{1}=F(\cdot, 1)$ are smooth and have only transversal periodic points. Suppose $d$ is odd, $\mathrm{PO}\left(f_{0}, d\right)$ contains only orbits of even Morse index, and $\mathrm{PO}\left(f_{1}, d\right)$ contains only orbits of odd Morse index. If $\gamma \in$ $\mathrm{PO}\left(f_{0}, d\right) \cup \mathrm{PO}\left(f_{1}, d\right)$ has least period $p$, then for each $k \geq 0$ there is $\gamma_{k} \in \mathrm{PO}\left(f_{0}, d\right) \cup$ $\mathrm{PO}\left(f_{1}, d\right)$ with least period $2^{k} p$ which is connected to $\gamma$ via $F$.

Note that $M$ is not necessarily compact in this theorem. This result was inspired by the main result of Alligood and Yorke $[\mathbf{A Y}]$ on period doubling in the creation of horseshoes and, to a certain extent, is a generalization of it. The hypotheses of Theorem B above are much more general and, consequently, the conclusions are necessarily weaker-in particular, one cannot assert the existence of sinks of period $2^{k} p$ under these hy potheses. However, in $\S 4$ we show how to derive the very beautiful result of Alligood and Yorke from this theorem.

As a final example we show how homological data can figure in the hypothesis. 
THEOREM C. Let $f: M \rightarrow M$ be a smooth map of a compact manifold with only transversal periodic points, and suppose that all eigenvalues of $f_{*}: H_{*}(M ; R) \rightarrow$ $H_{*}(M ; R)$ are $n$th roots of unity for some $n>0$. If $d$ is odd and does not divide $n$ and all orbits in $\mathrm{PO}(f, d)$ have Morse index of the same parity, then for each $\gamma \in \mathrm{PO}(f, d)$ of least period $p$ and each $k \geq 0$ there is a twisted periodic orbit of $f$ with least period $2^{k} p$.

1. The Lefschetz trace formula. The Lefschetz trace formula assigns an index (the Lefschetz number) to isolated fixed points of a self-map of a compact space and equates the sum of these numbers to homological invariants of the map. We make this more precise in a setting which is far from the greatest possible generality, but appropriate for our needs.

If $f: M \rightarrow M$ is a smooth map and $x \in M$ is a transversal fixed point then the Lefschetz number of $x$ with respect to $f$ is given by

$$
L(x, f)=\operatorname{sgn} \operatorname{det}\left(I-D_{x} f\right) .
$$

Note that the hypothesis that $x$ is transversal implies that $L(x, f)= \pm 1$ (and in fact this formula is not valid in general if $x$ is not transversal). We also observe that $L\left(x, f^{n}\right)$ may well differ from $L(x, f)$, so we will usually want the hypothesis that $x$ is a transversal fixed point of $f^{n}$ for all $n \geq 1$.

(1.1) Theorem (Lefschetz TRACE FORMula). Let $f: M^{n} \rightarrow M^{n}$ be a smooth map of a compact manifold with only transversal fixed points. Then

$$
\sum_{x \in \mathrm{Fix}(f)} L(x, f)=\sum_{k=0}^{n}(-1)^{k} \operatorname{tr} f_{* k}
$$

where $f_{* k}: H_{k}(M ; Q) \rightarrow H_{k}(M ; Q)$ is the map on $k$-dimensional rational homology induced by $f$.

For a proof see $[\mathbf{D}]$. It is important to describe $L(x, f)$ in terms of geometric properties of $D_{x} f$.

(1.2) Proposition. If $x$ is a transversal fixed point of a smooth map $f: M \rightarrow$ $M$ then

$$
L(x, f)=\Delta(x)(-1)^{u(x)},
$$

where $u(x)$ is the Morse index, i.e. the dimension of the subspace $E^{u} \subset T_{x} M$ corresponding to eigenvalues of $D_{x} f$ with asbolute value greater than one; and $\Delta(x)=1$ if $D_{x} f: E^{u} \rightarrow E^{u}$ preserves orientation, and -1 otherwise.

We are interested in periodic orbits $\gamma$ as well as fixed points. Hence if $\gamma$ is an orbit of $f$ with least period $p(\gamma)$ we define its Morse index to be the Morse index of $x \in \gamma$ for the map $f^{p(\gamma)}$ (for which it is a fixed point). This is easily seen to be independent of the choice of $x$. We also define $\Delta(\gamma)$ to be 1 if $D_{x} f^{p(\gamma)}: E^{u} \rightarrow E^{u}$ preserves orientation, and -1 otherwise. We call $\gamma$ twisted if $\Delta(\gamma)=-1$ and untwisted if $\Delta(\gamma)=1$.

Since there is a Lefschetz trace formula for each of the maps $f^{n}, n \geq 1$, we need a bookkeeping device to keep track of this infinite collection of equalities. The device we use is the Lefschetz zeta function $Z(f)$, defined by

$$
Z(f)=\exp \left(\sum_{m=1}^{\infty} \frac{L\left(f^{m}\right) t^{m}}{m}\right),
$$


where

$$
L\left(f^{m}\right)=\sum_{x \in \operatorname{Fix}\left(f^{m}\right)} L\left(x, f^{m}\right),
$$

if the fixed points of $f^{m}$ are isolated. It is quite easy to show from this expression and the Lefschetz trace formula that

$$
Z(f)=\prod_{k} \operatorname{det}\left(I-f_{* k} t\right)^{(--1)^{k+1}}
$$

where $f_{* k}: H_{k}(M ; Q) \rightarrow H_{k}(M ; Q)$ is the map induced by $f$ (see $\left.[\mathbf{F}]\right)$.

However, for our purposes the following expression for $Z(f)$ as an infinite product is equally important.

(1.3) THEOREM. If $f: M \rightarrow M$ has only transversal periodic points and $M$ is compact then, as formal power series,

$$
Z(f)=\prod_{\gamma \in \mathrm{PO}} A(\gamma)
$$

where $\mathrm{PO}$ is the set of periodic orbits of $f$,

$$
A(\gamma)= \begin{cases}1-\Delta(\gamma) t^{p(\gamma)} & \text { if the Morse index of } \gamma \text { is odd } \\ 1 /\left(1-\Delta(\gamma) t^{p(\gamma)}\right) & \text { otherwise, }\end{cases}
$$

$p(\gamma)$ is the least period of $\gamma$, and $\Delta(\gamma)$ is as defined above.

This theorem can be found as (5.19) of $[\mathbf{F}]$ with the slightly stronger hypothesis that the period points are hyperbolic. However the proof given there is equally valid for the result here so we do not repeat it.

(1.4) REMARKS. (a) Notice that simply knowing that $Z(f)$ depends only on the homotopy class of $f$ allows us to compute it in many cases without any homology. For example any $f: D^{n} \rightarrow D^{n}$ is homotopic to the constant map $g: D^{n} \rightarrow D^{n}$ given by $g(x)=0$ for all $x$. Since $g$ has only one periodic point, 0 , which is fixed and has Morse index 0 we conclude

$$
Z(f)=Z(g)=\frac{1}{1-t}=\sum_{m=0}^{\infty} t^{m}
$$

(b) In general the expression for $Z(f)$ given in (1.3) is an infinite product. However since all the periodic points are transversal there are only finitely many of any period $p$. Hence there are finitely many terms of the form $\left(1 \pm t^{p}\right)$ and of the form

$$
\frac{1}{1 \pm t^{p}}=\sum_{m=0}^{\infty}\left( \pm t^{p}\right)^{m}
$$

So as a formal power series this infinite product makes sense, since for each $n$ only finitely many terms contribute to the coefficient of $t^{n}$.

(c) The expression for $Z(f)$ as an infinite product of the $A(\gamma)$ can be considerably generalized. In particular, D. Fried $[\mathbf{F r}]$ shows that if $g$ is smooth with isolated (but not necessarily transversal) fixed points of $f^{n}, n \geq 1$, then $Z(f)=\prod_{\gamma \in \mathrm{PO}} A(\gamma)$, where each $A(\gamma)$ can be written as a finite product of terms of the form $\left(1 \pm t^{p}\right)^{ \pm 1}$. 
2. Formal power series. In this section we develop some elementary facts about formal power series with integer coefficients. We denote by $G$ the set $1+$ $t Z[[t]]$, i.e. $G$ is the set of formal power series with constant term 1 and integer coefficients. It is easy to see that $G$ is a group under multiplication since one can solve recursively for the coefficients of $g^{-1}$ for any $g \in G$.

(2.1) Proposition. If $g \in G$ then $g$ can be written uniquely as an infinite product

$$
g=\prod_{n=1}^{\infty}\left(1-\Delta_{n} t^{n}\right)^{a(n)}
$$

where $a(n) \geq 0$ and $\Delta_{n}$ is 1 or -1 .

This means that for each $g \in G$ there are unique choices for $a(n)$ and for $\Delta_{n}$ if $a(n) \neq 0$ such that the expression above is valid.

PROOF. Given $g=\sum c_{i} t^{i}$ it is easy to see one can solve recursively for the $\Delta_{n}$ and $a(n)$. We need only observe that $c_{n}$, the coefficient of $t^{n}$, is clearly equal to $-n \Delta_{n} a(n)$ plus terms involving $\Delta_{k}$ and $a(k), k<n$. Thus, since $a(n) \geq 0$ and $\Delta_{n}= \pm 1$, we can recursively solve for both of them. Q.E.D.

(2.2) Definition. For a fixed odd number $d>0$ let $G_{d}$ denote the subset of $G$ whose elements, when expanded as the product (2.1), satisfy $a(n)=0$ if $n \neq 2^{i} d$.

(2.3) Proposition. Each $G_{d}$ is a subgroup of $G$ and the group $G$ is isomorphic to the direct product $\prod_{d \text { odd }} G_{d}$.

Proof. The key observation here is that

$$
\left(1-t^{n}\right)^{-1}=\left(1+t^{n}\right)\left(1+t^{2 n}\right)\left(1+t^{4 n}\right)\left(1+t^{8 n}\right) \cdots,
$$

from which it follows that $G_{d}$ is closed under taking inverses and hence a subgroup. Clearly $G_{d} \cap G_{d^{\prime}}=1$ if $d \neq d^{\prime}$. From the uniqueness part of $(2.1)$ it is clear that the map $\prod_{d \text { odd }} G_{d} \rightarrow G$, given by $\left(g_{1}, g_{3}, g_{5}, \ldots\right) \mapsto g_{1} g_{3} g_{5} \cdots$, is an isomorphism. Q.E.D.

(2.4) LEMMA. If $d>0$ is odd and

$$
\prod_{n=0}^{\infty}\left(1-t^{2^{n} d}\right)^{a(n)} \prod_{n=0}^{\infty}\left(1+t^{2^{n} d}\right)^{b(n)}=1,
$$

then $b(n)=\sum_{i \leq n} a(i)$. If the equality above is replaced by congruence $\bmod t^{N}$, the result holds for $n<N$.

Proof. For $n=0$ it is clear that if $b(0) \neq a(0)$ then the coefficient of $t^{d}$ in the product will not be 0 .

We inductively assume the result for $n$ and prove it for $n+1$. Since

$$
\left(1-t^{p}\right)\left(1+t^{p}\right)\left(1+t^{2 p}\right) \cdots\left(1+t^{2^{n} p}\right)=1-t^{2^{n+1} p}
$$

we have by the induction hypothesis that the product of all terms with exponents $2^{i} d, i \leq n$, is equal to $\left(1-t^{2^{n+1}} d\right)^{b(n)}$. Hence we must have $b(n+1)=a(n+1)+b(n)$ or else the coefficient of $t$ with exponent $2^{n+1} d$ will not be zero. The case with congruence $\bmod t^{N}$ is proved similarly. Q.E.D.

(2.5) REMARK. It is clear from the proof of (2.3) that if $d$ is odd and $\rho_{d}: G \rightarrow G_{d}$ is projection onto the $d$ th factor then if $g=\prod_{n=1}^{\infty}\left(1-\Delta_{n} t^{n}\right)^{a(n)}$ as in (2.1), $\rho_{d}(g)=\prod_{n \in K}\left(1-\Delta_{n} t^{n}\right)^{a(n)}$, where $K=\left\{n \mid n=2^{k} d\right.$ for some $\left.k \geq 0\right\}$. 
3. The main theorem and proofs. According to (2.3) the group $G$ of formal power series with constant term 1 is isomorphic to the direct product $\prod_{d \text { odd }} G_{d}$. Let $\rho_{d}: G \rightarrow G_{d}$ be projection onto the fact $G_{d}$ as descirbed in (2.5).

(3.1) Definition. Define the $d$-component of the Lefschetz zeta function $Z(f)$ to be $\rho_{d}(Z(f))$ and denote it by $Z(f, d)$. It is only defined when $d$ is odd and $\geq 1$.

We can now state our main result.

(3.2) Theorem. Suppose $f: M \rightarrow M$ is a smooth map of a compact manifold and has only transversal periodic points. Then, as formal power series,

$$
Z(f, d)=\prod_{\gamma \in \operatorname{PO}(f, d)} A(\gamma),
$$

where $\mathrm{PO}(f, d)$ is the set of orbits whose periods are $2^{k} d$ for some $k \geq 0$.

Recall from (1.3) that $A(\gamma)$ is defined to be $\left(1-\Delta(\gamma) t^{p(\gamma)}\right)$ if the Morse index of $\gamma$ is odd, and the reciprocal of this otherwise, while $p(\gamma)$ is the least period, and $\Delta(\gamma)$ is -1 if $\gamma$ is twisted and 1 if it is untwisted.

PROOF. By definition $Z(f, d)=\rho_{d}(Z(f))$, where $\rho_{d}: G \rightarrow G_{d}$ is projection onto the $G_{d}$ component. Clearly from $(2.5)$,

$$
\rho_{d}\left(\prod_{\gamma \in \mathrm{PO}} A(\gamma)\right)=\prod_{\gamma \in \operatorname{PO}(f, d)} A(\gamma),
$$

so the result follows immediately from (1.3). Q.E.D.

(3.3) Proof of Theorem A. Consider first the case when $\mathrm{PO}(f, d)$ contains no orbits of even Morse index. Then for $\eta \in \operatorname{PO}(f, d)$ we have

$$
A(\eta)=\left(1-\Delta(\eta) t^{p(\eta)}\right),
$$

where $p(\eta)=2^{k} d$ for some $k \geq 0$. Also $Z(f)=1 / 1-t=(1+t)\left(1+t^{2}\right)\left(1+t^{4}\right) \cdots$ $(\sec (1.2(\mathrm{a})))$ so if $d>1, Z(f, d)=1$. We conclude that

$$
\prod_{\eta \in \operatorname{PO}(f, d)} A(\eta)=1
$$

and since $A(\gamma)=\left(1-\Delta_{\gamma} t^{p}\right)$, it follows from (2.4) that for each $k \geq 0$ there is an $\eta \in \mathrm{PO}(f, d)$ with period $2^{k} p$ and $\Delta(\eta)=-1$, i.e. a twisted periodic orbit of period $2^{k} p$. If $d=1$ then

$$
(1-t) \prod_{\eta \in \operatorname{PO}(f, d)} A(\eta)=1
$$

and we obtain the same conclusion. If $\mathrm{PO}(f, d)$ contains no orbits of odd index, the same argument using $A(\gamma)^{-1}$ in place of $A(\gamma)$ gives the desired result. Q.E.D.

Suppose now that $F: M \times I \rightarrow M$ has bounded periodic set and let $f_{\mu}=F(\cdot, \mu)$. We suppose also that for each $n$ the sets $\operatorname{Fix}\left(f_{0}, n\right)$ and $\operatorname{Fix}\left(f_{1}, n\right)$ have finitely many components (in the application to the proof of Theorem $\mathrm{B}$ they will be finite). For some integer $N>0$ let $C$ be a union of $\varepsilon$-components of $\operatorname{Fix}(\bar{F}, N)$ where $\bar{F}(x, \mu)=(F(x, \mu), \mu)$. Recall that two points $x, y \in \operatorname{Fix}(\bar{F}, N)$ are in the same $\varepsilon$-component if there are points $x_{0}=x, x_{1}, \ldots, x_{n}=y$, in $\operatorname{Fix}(\bar{F}, N)$, such that $d\left(x_{1}, x_{i+1}\right)<\varepsilon$. We choose $\varepsilon$ sufficiently small such that each $\varepsilon$-component contains at most one of the components of $\operatorname{Fix}\left(f_{0}, N\right)$ and at most one of the components 
of $\operatorname{Fix}\left(f_{1}, N\right)$. The point of all this is that $\operatorname{Fix}(\bar{F}, N)$ may have infinitely many components, in which case there will be components which are not isolated. There are always finitely many $\varepsilon$-components in a compact space, however, and each of them is isolated.

For $\mu \in[0,1]$ let $C_{\mu}=\{x \in M \mid(x, \mu) \in C\}$ and let $\operatorname{PC}\left(f_{\mu}, d\right)$ be the set of periodic orbits of $f_{\mu}$ which have period $2^{k} d$ for some $k$ and which contain a point of $C_{\mu}$.

(3.4) Proposition. Suppose $F: M \times I \rightarrow M$ has bounded periodic set, $d$ is odd, and $\mathrm{PC}\left(f_{\mu}, d\right)$ is as above. If, for $i=0,1$, the set $C_{i}$ consists of transversal periodic points for $f_{i}$ then the power series

$$
\prod_{\gamma \in \operatorname{PC}\left(f_{i}, d\right)} A(\gamma), \quad i=0,1,
$$

are equal when truncated at $t^{N}$.

Proof. We consider the power series

$$
\alpha_{\mu}=\prod_{\gamma \in \operatorname{PC}\left(f_{\mu}\right)} A(\gamma)=\prod_{d \text { odd }}\left(\prod_{\gamma \in \operatorname{PC}\left(f_{\mu}, d\right)} A(\gamma)\right),
$$

which is defined for $\mu=0$ or 1 , where $\mathrm{PC}\left(f_{\mu}\right)$ is the set of all periodic orbits of $f_{\mu}$ which contain a point $C_{\mu}$. One can define the Lefschetz index $L(X, f)$ of an arbitrary isolated compact subset $X$ of $\operatorname{Fix}(f)$ and it shares the same homotopy properties as the index of a point (see $[\mathbf{D}]$ ). We wish now to consider the numbers $L\left(C_{\mu}, f_{\mu}^{m}\right)$. The coefficient of $t^{i}, i<N$, in $\alpha_{\mu}$ is the same as the coefficient of $t^{i}$ in the series

$$
\beta_{\mu}=\exp \left(\sum_{m=1}^{\infty} \frac{L\left(C_{\mu}, f_{\mu}^{m}\right) t^{m}}{m}\right) .
$$

The proof is the same as the proof of (1.3) and is an easy consequence of the fact that

$$
\left(1-\Delta t^{p}\right)^{-1}=\exp \sum_{m=1}^{\infty} \frac{\left(\Delta t^{p}\right)^{m}}{m} .
$$

We note that $\beta_{\mu}$ can be defined for all $\mu \in[0,1]$, and if $\left[\beta_{\mu}\right]_{N}$ is $\beta_{\mu}$ truncated at $t^{N}$ then it depends only on the numbers $L\left(C_{\mu}, f_{\mu}^{m}\right), 1 \leq m \leq N$, which are independent of $\mu$ (see $[\mathbf{D}]$ ). It follows that $\left[\alpha_{0}\right]_{N}=\left[\alpha_{1}\right]_{N}$.

From this and $(2.5)$ we know $\left[\rho_{d}\left(\alpha_{0}\right)\right]_{N}=\left[\rho_{d}\left(\alpha_{1}\right)\right]_{N}$, where $\rho_{d}: G \rightarrow G_{d}$ is projection. But

$$
\rho_{d}\left(\alpha_{\mu}\right)=\prod_{\gamma \in \operatorname{PC}\left(f_{\mu}, d\right)} A(\gamma), \quad \mu=0,1,
$$

by (2.5) so we have the desired result. Q.E.D.

(3.5) Proof of Theorem B. Let $N=2^{k} p$, let $C$ be the union of all components of $\operatorname{Fix}(\bar{F}, N)$ which contain points of $\gamma$, and recall that $C_{\mu}=\{x \in M \mid(x, \mu) \in$ $C\}$.

By (3.4) the power series

are equal when truncated at $t^{N}$.

$$
\prod_{\eta \in \operatorname{PC}\left(f_{\mu}, d\right)} A(\eta), \quad \mu=0,1
$$


If $\eta \in \mathrm{PC}\left(f_{\mu}, d\right)$ and $\mu=1$ then $A(\eta)=\left(1-\Delta(\eta) t^{p(\eta)}\right)$, while if $\mu=0, A(\eta)^{-1}$ has this form because of our hypothesis on the parity of the Morse index.

Since our remarks above imply

$$
\prod_{\mu=1} A(\eta) \prod_{\mu=0} A(\eta)^{-1} \equiv 1 \quad\left(\bmod t^{N}\right)
$$

(the products being over all $\eta \in \mathrm{PC}\left(f_{\mu}, d\right)$ ), the existence of $\gamma \in \mathrm{PC}\left(f_{\mu}, d\right)$ for $\mu=0$ or 1 , together with $(2.4)$, gives the desired result. Q.E.D.

(3.6) Proof OF THEOREM C. The hypothesis on the eigenvalues of $f_{*}$ : $H_{*}(M ; R) \rightarrow H_{*}(M ; R)$, together with the fact that

$$
Z(f)=\prod_{k} \operatorname{det}\left(I-f_{* k} t\right)^{(-1)^{k+1}}
$$

makes it easy to see that $Z(f)$ can be written in the form

$$
\prod_{i}\left(1-t^{p(i)}\right) / \prod_{j}\left(1-t^{q(j)}\right)
$$

where each $p(i)$ and $q(j)$ is a divisor of $n$. In fact any polynomial whose roots are $n$th roots of unity can be written in this form.

It follows from $(2.5)$ that $Z(f, d)=\rho_{d}(Z(f))=1$ if $d$ does not divide $n$. With this fact the remainder of the proof is identical to the proof of Theorem A. Q.E.D.

4. Area preserving and area contracting maps. In this section we show how one can easily derive the result of Alligood and Yorke in $[\mathbf{A Y}]$ from our Theorem B. This gives a new proof of this very important theorem. We state the result with a somewhat less restrictive hypothesis and consider only dimension two, for simplicity, but this is essentially the main theorem of $[\mathbf{A Y}]$.

(4.1) Theorem (Alligood-YoRKe [AY]). Let $F: R^{2} \times I \rightarrow R^{2}$ be a oneparameter family of diffeomorphisms with bounded periodic set. Suppose $f_{\mu}=$ $F(\cdot, \mu)$ and $f_{0}$ has no periodic points, while $f_{1}$ has only periodic saddles, including at least one, $\gamma$, of period $p$.

(1) If $f_{\mu}$ is area preserving for $\mu \in[0,1]$, then for each $k \geq 0$ there exists a parameter value $\lambda(k)$ and an elliptic periodic orbit $\gamma_{k}$ for $f_{\lambda(k)}$ with irrational rotation number and least period $2^{k}$ p. Also $\gamma_{k}$ is connected to $\gamma$ via $F$.

(2) If $f_{\mu}$ is uniformly area contracting for $\mu \in[0,1]$, then for each $k \geq 0$ there exists a periodic sink $\gamma_{k}$ of $f_{g(k)}$ with least period $2^{k} p$ which is connected to $\gamma$ via $F$.

PROOF. We first consider (2). Let $G: R^{2} \times I \rightarrow R^{2}$ be a generic one-parameter family of diffeomorphisms approximating $F$ uniformly on some compact neighborhood $K$ of all periodic points. In this family only saddle-node and period doubling bifurcations occur, say for periods up to $4^{k} p$ (see $[\mathbf{B r}]$ ). Applying Theorem B to $G$ it is clear that if $C$ is the component $\operatorname{Fix}\left(\bar{G}, 2^{k} p\right)$ which contains $(x, 1)$ for some $x \in \gamma$, we can draw an arc $\alpha(t)$ in $C$ with $\alpha(0)=(x, 1)$ and $\alpha(1)=(y, 1)$, where $y$ is a periodic saddle of period $2^{k} p$ for $f_{1}$. The arc $\alpha(t)$ goes through various bifurcations of period-doubling or saddle-node type. All of these involve a saddle and a 
sink since we assume $G$ to be a sufficiently good approximation to $F$ that there are no sources.

Define $b \in[0,1]$ to be the smallest $t$ such that there is a period doubling at $\alpha(t)$ from period $2^{k} p$ to $2^{k+1} p$ and define $a$ to be the largest $t<b$ such that there is a period-doubling at $\alpha(t)$ from period $2^{k-1} p$ to $2^{k} p$ (in case $k=0$ and there are no such points choose $a$ so $\alpha(a)$ is the first bifurcation point).

Now for $t \in(a, b], \alpha(t)$ has least period $m=2^{k} p$ and the eigenvalues for the derivative of the $m$ th iterate of the map include 1 at $\alpha(a)$ and -1 at $\alpha(b)$. Since all our maps are diffeomorphisms there must be a $c \in(a, b)$ where the eigenvalues at $\alpha(c)$ are complex. Since the product of the two eigenvalues, $\lambda$ and $\bar{\lambda}$, is less than $k<1$ (because of uniform area contraction), we conclude $|\lambda|=|\bar{\lambda}|<\sqrt{k}<1$. Thus for $G$ there is a parameter value $\mu$ for which $G(\cdot, \mu)$ has a sink $x$ of period $2^{k} p$ with eigenvalues $\lambda, \bar{\lambda}$ satisfying $|\lambda|=|\bar{\lambda}|<\sqrt{k}<1$.

If we choose a sequence $G_{i}$ of generic approximations converging uniformly to $F$ on $K$ then for some parameter $\mu_{i}$ each has a sink $x_{i} \in K$ of least period $2^{k} p=m$ and eigenvalues $\lambda_{i}, \bar{\lambda}_{i}$ with $\left|\lambda_{i}\right|=\left|\bar{\lambda}_{i}\right|<\sqrt{k}$. A subsequence of $\left\{x_{i}\right\}$ and $\left\{\mu_{i}\right\}$ converges to $y \in K$ and $\mu \in I$, respectively, and $F^{m}(y, \mu)=y$. Also $y$ will have eigenvalues of absolute value $<\sqrt{k}$ so will be a sink. Since a sink cannot be a bifurcation point, $m$ must be its least period.

The proof of the area preserving case $(1)$ is essentially the same. There one chooses $c$ so that the eigenvalues at $\alpha(c)$ are not only complex but of the form $\lambda=r e^{2 \pi i \theta}$ for some real $r>0$ and a fixed irrational $\theta$. If the same $\theta$ is used for each $G_{i}$, the limiting elliptic point will have rotation number $\theta$. Q.E.D.

(4.2) EXAMPLE. Let $F(x, y)=\left(A+B y-x^{2}, y\right)$. This is the so-called Henon map, which is area preserving for $B=1$ and area contracting if $0<B<1$. In [DN] Devaney and Nitecki prove that this map with $B$ fixed and $A$ as parameter satisfies the hypothesis of the Alligood-Yorke theorem above. In particular, for $A<-(1+|B|)^{2} / 4$ there are no periodic points, and for $A>(5+2 \sqrt{5})(1+|B|)^{2} / 4$ all periodic points are saddles and there are points of every period.

\section{REFERENCES}

[AY] K. Alligood and J. Yorke, Cascades of period doubling bifurcations: A prerequisite for horseshoes. Bull. Amer. Math. Soc. (to appear).

[B] P. Blanchard, Invariants of the NPT isotpy classes of Morse Smale diffeomorphism of surfaces, Dukc Math. J. 47 (1980), 33-46.

[Br] P. Brunovsky, On one-parameter families of diffeomorphisms. I, II, Comment. Math. Univ. Carolinae 11 (1970), 559-582; 12 (1971), 765784.

[DN] R. Devaney and Z. Nitecki, Shift automorphisms in the Henon mapping, Comment. Math. Phys. 67 (1979), $137-146$.

[D] A Dold, Fixed point index and fuxed point theorems for Euclidean neighborhood retracts, Topology 4 (1965), 1-8.

[F] J. Franks, Homology and dymamical systems, CBMS Regional Conf. Ser. in Math., no. 49, Amer. Math. Soc., Providence, R.I., 1982.

[Fr] D. Fried, Periodic points and twisted coefficients (to appear).

Department of Mathematics. Northwestern University, EVANSTON. Illinois 60201 